\title{
A PROGRAM IN MEDIUM-ENERGY NUCLEAR PHYSICS
}

Prof. B.L. Berman, Principal Investigator

Assoc. Prof. K.S. Dhuga, Co-Principal Investigator

\author{
Center for Nuclear Studies \\ Department of Physics \\ The George Washington University \\ Washington, DC 20052
}

PROGRESS REPORT--SEPTEMBER 1, 1992-JUNE 30, 1993

\section{Overview}

This report reviews progress on our nuclear-physics program for the last ten months, and includes as well copies of our publications and other reports for that time period. The structure of this report follows that of our 1992 Progress Report: Sec. II outlines our research activities aimed at future experiments at CEBAF, NIKHEF, and Bates; Sec. III gives results of our recent research activities at NIKHEF, LAMPF, and elsewhere; Sec. IV provides an update of our laboratory activities at GWU, including those at our new Nuclear Detector Laboratory at our Virginia Campus; and Sec. V is a list of our publications, proposals, and other reports. Copies of those on medium-energy nuclear physics are reproduced in the Appendix.

The highlight of the year has been the approval by the NIKHEF and CEBAF PACs of all three of the proposals we have submitted. These are "Recoil Polarization of the Neutron in the Reactions "He(e,e'n) and "He(e,e'n)," NIKHEF Proposal 93-09 (K.S. Dhuga, spokesperson), "Photoreactions on ${ }^{3} \mathrm{He}$, CEBAF Proposal 93-044 (B.L. Berman, co-spokesperson), and "Photoabsorption and Photofission of Nuclei," CEBAF Proposal 93-019 (B.L. Berman, cospokesperson). The NIKHEF experiment involves the use of the High-Acceptance Recoil Polarimeter (HARP) for detection and measurement of the polarization of the emitted neutron. We, together with our colleagues at Grenoble, are responsible for the design and construction of the wire chambers for this device; we have largely completed the design phase this past year. The CEBAF experiments involve the use of the Hall-B Photon Tagger for production of the monochromatic photon beam. We (all of us at GWU, but particularly the NSF-sponsored group led by W.J. Briscoe) are responsible for the 432 -scintillator focal-plane detector array for this device; again, most of the design work and some prototype testing have been completed this past year. In addition, we have continued to make progress on data analysis and publication of results of previous measurements at Bates, LAMPF, and NIKHEF, and we also are planning for new experimental runs at these laboratories in the coming year.

DISTRIBUTION OF THIS DOCUMENT IS UNLIMITED
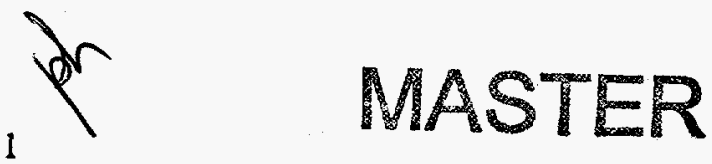


\section{DISCLAIMER}

This report was prepared as an account of work sponsored by an agency of the United States Government. Neither the United States Government nor any agency thereof, nor any of their employees, makes any warranty, express or implied, or assumes any legal liability or responsibility for the accuracy, completeness, or usefulness of any information, apparatus, product, or process disclosed, or represents that its use would not infringe privately owned rights. Reference herein to any specific commercial product, process, or service by trade name, trademark, manufacturer, or otherwise does not necessarily constitute or imply its endorsement, recommendation, or favoring by the United States Government or any agency thereof. The views and opinions of authors expressed herein do not necessarily state or reflect those of the United States Government or any agency thereof. 


\section{DISCLAIMER}

Portions of this document may be illegible electronic image products. Images are produced from the best available original document. 


\section{Progress on Our Current Research Program}

In the following paragraphs, we give some details of the various components of our current research program. A concise listing is provided by Tables I and II, which give the motivation and status of the experiments planned for the coming year and for future years, respectively.

\section{A. Measurements with Real Photons at CEBAF}

A large part of our effort this past year has gone into the planning and preparation for experiments with real photons at the CEBAF Large-Acceptance Spectrometer (CLAS). The present PI spent most of his sabbatical year (1991-92) at CEBAF, and serves as chair of the RealPhoton Physics Working Group of the CLAS Collaboration. The two proposals that we submitted this year for Hall B have been approved. We also are part of the collaboration for another proposal that was approved by the $1993 \mathrm{PAC}$, as well as for two others that were approved by the previous CEBAF PAC in 1991. And although our primary interests at CEBAF are centered on the tagged-photon beam and CLAS in Hall B, we also plan to become involved in the more distant future in experiments in Hall A (see Sec. 6 below).

\section{1) Photoreactions on ${ }^{3} \mathrm{He}$}

This proposal was prepared in collaboration with groups at Genoa and Saclay; the cospokespersons are B.L. Berman, P. Corvisiero, and G. Audit. It consists of three major parts. Three-body-force effects, primarily our responsibility, will be studied via the $(\gamma, 3 \mathrm{~N})$ and $(\gamma, \Delta \mathrm{NN})$ reaction channels. Modification of the properties of nucleon resonances, principally the $D_{13}$ and $F_{15}$, in the ${ }^{3} \mathrm{He}$ nucleus, primarily the responsibility of the Genoa group, will be studied via the $(\gamma, \pi 3 \mathrm{~N})$ and $(\gamma, 2 \pi 3 \mathrm{~N})$ channels. The probability for a small (pre-existing) $\Delta \mathrm{NN}$ component in the ${ }^{3} \mathrm{He}$ ground-state wave function, primarily the responsibility of the Saclay group, will be studied via direct $\Delta^{++}$knockout [the $\left(\gamma, \mathrm{p} \pi^{+}\right)$and $\left(\gamma, \mathrm{p} \pi^{+} \mathrm{n}\right)$ channels]. The instrumentation contributions of the three groups are the photon-tagger focal-plane detector array, the large-angle shower counter, and the cryogenic target assembly, respectively.

\section{2) Photoabsorption and Photofission of Nuclei}

This proposal was prepared in collaboration with the photonuclear group at Frascati (cospokespersons, N. Bianchi and B.L. Berman), on total photon absorption by nuclei. For a variety of nuclei, including $\mathrm{C}, \mathrm{Al}, \mathrm{Cu}, \mathrm{Sn}$, and $\mathrm{Pb}$, the total-hadronic detection method will be used. The 
$\mathrm{NaI}$ and lead-glass detectors for this part of the experiment will be contributed by the Frascati and Genoa groups. For the heaviest nuclei, the most convenient way to accomplish this is via the photofission channel, which for these nuclei is expected to constitute nearly all, if not all, of the total photon absorption strength. We shall study the photofission of ${ }^{238} \mathrm{U},{ }^{237} \mathrm{~Np}$, and ${ }^{232} \mathrm{Th}$. The salient points of this part of the proposal had been given in CLAS Note 92-010 (B.L. Berman et al.). We shall use parallel-plate avalanche detectors (PPADs), designed at GWU and built in collaboration with colleagues at São Paulo and Novosibirsk, for detection of the fission fragments.

\section{3) Photoproduction of Vector Mesons at High $t$}

The main goal of this proposal [co-spokespersons, J.-M. Laget (Saclay) and M. Anghinolfi (Genoa)] is to study the photoproduction of $\phi$ mesons at large $t$ on the proton and ${ }^{3} \mathrm{He}$. This reaction is expected to proceed via the exchange of two gluons, which provides us with a unique way to study hidden-color components (or more generally correlations between quarks) in hadronic matter. The signature of the $\phi$ will be its decay into two kaons. At the same time, the two-pion decay channels will be measured, and the consequent data on the production of $\rho$ and $\omega$ mesons, as well as $\pi \Delta$ pairs, will enlarge our knowledge of other hard quark-rearrangement mechanisms. The $\mathrm{K}^{+} \Lambda$ channel also will be clearly identified.

\section{4) Vector Meson Production from the Proton and Deuteron}

The co-spokespersons for this 1993 Letter of Intent are B.L. Berman, W.J. Briscoe, and R.R. Whitney (CEBAF). The experiment will employ a linearly polarized tagged photon beam with the CLAS. The aim of the study is to map out the cross section for $\rho$-meson production as a function of $t$ in the region of 0.5 to $1.0(\mathrm{GeV} / \mathrm{c})^{2}$, and also to extract the spin-density matrix elements over this kinematic range. The chosen kinematic range is of considerable interest since the production process is thought to switch in this region from a purely diffractive one (i.e., hadronic in nature) to a bare or point-like one. Apart from preparing the physics proposal, we shall be involved in the fabrication and installation of the equipment for the production of the linearly polarized tagged photon beam (see Sec. 5 below).

\section{5) Tagged Coherent Bremsstrahlung}

A Monte-Carlo simulation program is being developed to model all aspects of both "normal" and coherent tagged bremsstrahlung produced with a diamond radiator at the Hall $\mathrm{B}$ photon tagger. In particular, the energy spectrum and polarization of the coherent bremsstrahlung will be studied in detail and compared with existing data. An alternative source for polarized 
tagged photons, using Compton backscattering from a high-power laser, has been submitted as a Letter of Intent to CEBAF by B.E. Norum (Univ. of Virginia); a decision on the implementation of this method has not yet been reached. The design for the tagger incorporates provisions for either of these methods for producing polarized tagged photons.

\section{6) The HARP at CEBAF}

The High-Acceptance Recoil Polarimeter (HARP) is a high-efficiency, large-solid-angle neutron detector and polarimeter being designed and constructed by a collaboration of six research groups in North America and Europe, including GWU. The initial objective is to to be able perform a program of (e,e'n) and (e,e'pn) measurements on few-body nuclei at energies up to $800 \mathrm{MeV}$ (incident electron energy) at NIKHEF, then to relocate to CEBAF to continue the research program in the 1-to- $6 \mathrm{GeV}$ range. An application for Hall A membership by the HARP Collaboration was approved last summer. Physics of interest includes the investigation of shortrange nucleon-nucleon correlations in few-body systems, the extraction of selected spindependent response functions, particularly in the region of the delta and Roper resonances where the interference terms are sensitive to the quadrupole deformation of the delta, and to possible longitudinal excitations of the Roper resonance.

\section{B. The HARP and (e,e'n) Measurements at NIKHEF}

We are now engaged in a program of $\left(e, e^{\prime} n\right)$ and $\left(e, e^{\prime} p n\right)$ measurements on few-body nuclei at NIKHEF. For most of these measurements we shall employ the HARP. For the construction of such a large-scale neutron detector, we have formed a collaboration with groups from the University of Utrecht (Netherlands), the Institute of Nuclear Physics at Grenoble (France), the University of Regina (Canada), the University of Maryland, and NIKHEF. The GWU participation in this collaboration is funded in part by the DOE University Research Instrumentation Program. The GWU spokesperson for this project is K.S. Dhuga; the overall HARP Collaboration Coordinator is T.S. Bauer.

\section{1) Neutron Recoil Polarization in the ${ }^{4} \mathrm{He}\left(e, e^{\prime} n\right)$ Reaction}

This proposal, for which the spokesperson is K.S. Dhuga, is to measure the normal component of the neutron polarization vector in parallel kinematics for values of $Q^{2}$ equal to 0.09 , 0.16 , and $0.23(\mathrm{GeV} / \mathrm{c})^{2}$. The normal component of the recoil polarization is predicted to be identically zero in the PWIA--it acquires a nonzero value entirely through final-state interactions 
(FSI). Its description therefore represents a stringent test of microscopic models that go beyond the PWIA. These models indicate that the polarization data will provide a quantitative measure of the role of meson-exchange currents, and, in particular, the two-step charge-exchange process (e,e'p) followed by $(p, n)$. The experiment is expected to run in 1995, and most likely will form the basis of a Ph.D dissertation for one our graduate students. With the aid of the GEANT and EGS computer codes, we are carrying out Monte-Carlo studies of the ${ }^{4} \mathrm{He}\left(e, e^{\prime} n\right)$ reaction under various geometrical arrangements of the detection system. The results will allow us to gauge the importance of some of the leading background processes that are likely to contribute to the extracted signal.

\section{2) The ${ }^{3} \mathrm{He}\left(\mathrm{e}, \mathrm{e}^{\prime} \mathrm{pn}\right)$ Reaction in the Region Beyond the Quasi-elastic Peak}

The aim of this approved (in 1991) proposal [spokesperson, T.S. Bauer (Univ. of Utrecht)] is to explore the energy, angle, and momentum dependence of the two-nucleon absorption cross section. The kinematics for this experiment have been chosen to enhance such two-nucleon correlations in the ${ }^{3} \mathrm{He}$ ground state as may exist.

\section{3) Longitudial/Transverse Separation of the ${ }^{4} \mathrm{He}\left(e, e^{\prime} n\right)$ Reaction}

This proposal to measure the longitudinal and transverse response functions for ${ }^{4} \mathrm{He}(e, e$ 'n) [spokesperson, T.S. Bauer, (Univ. Utrecht)], was approved by the 1991 NIKHEF PAC. Although the neutron-knockout reaction is expected to be sensitive to the effects of final-state interactions (FSI), almost no data exist for this reaction. Measurement of the quasifree neutronknockout reaction under kinematic conditions allowing a direct comparison with the protonknockout data will contribute to our understanding of the mechanism of electron-induced nucleon knockout as well as helping to determine the role of FSI, especially charge exchange. The experiment will be performed with the NIKHEF QDQ spectrometer and a neutron-time-of-flight detector in late 1993 or early 1994, before the construction of the HARP is completed.

\section{4) The High-Acceptance Recoil Polarimeter (HARP)}

The neutron detection system consists of a liquid hydrogen vessel, multiwire proportional chambers (MWPCs), and plastic scintillators, all enclosed in a lead-steel housing. The basic principle of operation is that of recoil detection; the recoil proton from an $n-p$ scattering event is tracked and its energy measured. The liquid hydrogen serves as the n-to-p converter, the MWPCs provide the track definition and angle information, and the $(E-\triangle E)$ scintillators are used to determine the type and energy of the recoil particle. The MWPCs and the scintillators are arranged symmetrically about the n-p converter either in the left-right or the up-down 
configuration. This feature not only allows measurements out of plane, but also allows the measurement of the polarization of the incident particle because of the asymmetry of the $n-p$ scattering process. A particle tagger (thin scintillator) in front of the HARP converter along with the particle identification capabilty of the $E-\Delta E$ scintillators will in principle allow simultaneous measurement of the (e,e'n) and (e,e'p) reactions on a given target nucleus. The requirements for the detector are an excellent signal-to-background ratio, relatively high neutron detection efficiency, and polarization information on the detected neutron. Each Member Institution of the HARP Collaboration is responsible for certain aspects of the HARP detector development. The joint task of the GWU and the Grenoble groups is the design, construction, testing, and final implementation (i.e., integration into the HARP detector) of the large MWPCs.

\section{5) Multiwire Proportional Chambers}

Presently, the MWPC project is in the blueprint phase, expected to be completed by the end of July. The construction phase is expected to commence in September. Much of the design, carried out by P.L. Cole and E. Voutier (Grenoble), has been driven by the requirements of spatial resolution, sensitivity to counting rates, the electronic readout system, and the overall cost. Some of the detailed design considerations are based upon the tracking chambers of the Fermilab fixedtarget experiment E706 and the MWPCs of the CERN Hyperon experiment WA89.

We have employed both detailed analytical calculations and the wire-chamber simulation code GARFIELD to set the basic chamber parameters. Each chamber will be composed of two measuring layers containing 448 sense wires and 8 guard wires. The anode wires will be $25-\mu \mathrm{m}$ diameter gold-plated tungsten-rhenium alloy. The cathode planes will be formed by a doublecoated gold/mylar sandwich. A $0.1-\mu \mathrm{m}$ gold coating will be vapor deposited on the $25-\mu \mathrm{m}$ thick mylar substrate. The cathode frames will be made out of Stesalit. This material was chosen for its high mechanical rigidity, nonconductivity, and the ease with which it can be machined to high precision over large lengths. Owing to external requirements, i.e., maximum acceptance for tracking protons in HARP, the MWPC module will be trapezoidal in shape, and can be contained within the area of a square of approximately $1.6 \mathrm{~m} \times 1.6 \mathrm{~m}$.

We shall use the facilities of Lab 6 at Fermilab for the manufacture and assembly of the HARP chambers. Most of the initial chamber tests involving such things as high voltage, gas mixtures, and chamber gains and leakage currents will be carried out at our own Nuclear Detector Laboratory. However, the full electronic readout tests will be conducted at Grenoble (or at NIKHEF) jointly by the GWU and the Grenoble groups, after the chambers have been transported to Europe. 


\section{6) $(n, p)$ Cross-Section Measurements at LAMPF in Support of HARP}

The critical element of the HARP detector is the neutron-to-proton converter. In order to decide whether liquid hydrogen, with all of its inherent difficulties and cost, must be used, or whether another material rich in hydrogen, such as polyethylene, is adequate for the job, a series of (n,p) cross-section measurements on carbon and polyethylene were carried out in 1991 and 1992 at the LAMPF/WNR facility. A preliminary analysis, by S.L. Rugari, of these data indicates that while the proton yield from the hydrogen in the polyethylene target appears to be large, it probably would not be cleanly distinguishable from the sizeable proton yield from the carbon contained in the target. A full analysis of all of our WNR data should be completed by the end of this summer.

\section{Nuclear-Structure Measurements at Bates}

An A-priority experiment left undone in our previous program of nuclear-structure measurements at the high-resolution electron-scattering spectrometer system (ELSSY) at the Bates Accelerator is inelastic electron scattering from collective states in ${ }^{21} \mathrm{Ne}$ and ${ }^{23} \mathrm{Na}$ [cospokespersons, B.L. Berman, D.M. Manley (Kent State Univ.), and R.A. Lindgren (Univ. of Virginia)]. These measurements were postponed because of the upgrade of the Bates facility to high duty factor. The physics argument for performing these measurements remains cogent, however, and we expect to obtain data on these nuclei in early 1994. We expect that Mr. D. Pang will obtain his Ph.D. thesis data from these measurements. 


\section{Results of Previous Experiments}

Over the past several years, we have performed a number of medium-energy nuclearphysics experiments with both electromagnetic and hadronic probes. A summary of the current status of those measurements that have not been completed and published in prior years is given in Tables III and IV, for few-body and shell-model nuclei, respectively. During the past year, we have made progress in analyzing data from these previous experiments and in publishing some of the results. Some details of this work are given below and in our previous progress reports.

\section{A. (e,e'x) Experiments at NIKHEF}

In our series of (e,ex) measurements on ${ }^{6} \mathrm{Li}$ and ${ }^{12} \mathrm{C}$ at NIKHEF, where $\mathrm{x}=\mathrm{d}, \alpha, \mathrm{t}$, or $\tau$, we have almost completed the analysis (mostly by J.P. Connelly) of the last in the series, namely, ${ }^{6} \mathrm{Li}\left(\mathrm{e}, \mathrm{e}^{\prime} \tau\right)$. These results, when combined with those for ${ }^{6} \mathrm{Li}\left(e, \mathrm{e}^{\prime} t\right)$, will elucidate the reaction mechanism, since the final-state interactions are the same for both mirror reactions. The results show that the $q$-dependence for the (e,e't) deviates markedly from that which would be expected from a system in which the trinucleon clusters were the same as they are in free space, while that for the $\left(e, e^{\prime} \tau\right)$ reaction does not. This striking contrast may imply a strong charge-exchange intermediate process between the trinucleon clusters. The ${ }^{6} \mathrm{Li}\left(e, e^{\prime} \alpha\right)$ results were published last year; the (e,e'd) results for both nuclei were published earlier as Letters, and a comprehensive paper covering all of the (e,e'd) results is nearly ready to be submitted for publication.

\section{B. Pion Scattering from ${ }^{3} \mathrm{H}$ and ${ }^{3} \mathrm{He}$ at LAMPF}

The analysis of the large-angle pion elastic-scattering data on ${ }^{3} \mathrm{H}$ and ${ }^{3} \mathrm{He}$ is now complete. Several papers, on various aspects of this work are being prepared for publication [by B.L. Berman, K.S. Dhuga, S.K. Matthews, and C. Pillai (LANL)]. Preliminary theoretical analysis of the large-angle data indicates that within the confines of the currently available models it is going to be very difficult to obtain a simultaneous description of both the cross sections and the superratios at 180 and $256 \mathrm{MeV}$. Recent theoretical calculations by Kamalov, Tiator, and Bennhold (the last a new faculty member of the GWU theory group) stress the need for 'exact' wave functions for the trinucleon system, resulting from Faddeev calculations with realistic nucleon-nucleon potentials. These authors also demonstrate that a second-order isospindependent potential is needed to achieve relatively good descriptions of the (180 and $256 \mathrm{MeV}$ ) cross-section data in the angular region forward of approximately $90^{\circ}$ in the center of mass. Even 
with higher-order corrections to the optical potential, however, the description of the superratio remains rather poor at both energies. Also, the absolute cross sections in the backward hemisphere are consistently predicted to be too small in these model calculations. Kamalov (from the Laboratory of Theoretical Physics, Dubna) will be visiting GWU this summer to continue these calculations in collaboration with Bennhold.

\section{Pion Scattering from Shell-Model Nuclei}

The large-angle pion elastic-scattering data for ${ }^{12} \mathrm{C}$ and ${ }^{16} \mathrm{O}$ have been analyzed (by $\mathrm{B}$. Park, New Mexico State Univ. and K.S. Dhuga), and a paper is being prepared for publication. Analysis of the remaining data, on ${ }^{28} \mathrm{Si}$ and ${ }^{40} \mathrm{Ca}$, is expected to be completed this summer. The high-energy (400 to $500 \mathrm{MeV}$ incident energy) pion elastic-scattering data on ${ }^{12} \mathrm{C},{ }^{16} \mathrm{O},{ }^{40} \mathrm{Ca}$, ${ }^{90} \mathrm{Zr}$, and ${ }^{208} \mathrm{~Pb}$ is currently being re-analyzed by C.L. Morris at LANL. Unfortunately, analysis of these data has been hindered by curious inconsistencies between $\pi^{+}$and $\pi^{-}$flux normalizations. Several normalization data points have been re-measured in order to resolve this normalization problem. We hope to carry out some preliminary theoretical analysis of these data in collaboration with Kamalov and Bennhold.

\section{Photonuclear Reactions}

The last of a long series of papers on the photonuclear reaction cross sections performed at Livermore and Melbourne has been completed; our long review paper on the isospin composition of the giant dipole resonance in light nuclei has been published. 


\section{The GWU Nuclear Detector Laboratories and Center for Nuclear Studies}

\section{A. The GWU Nuclear Detector Laboratories}

We now have moved into our new laboratory at the Virginia Campus. Our activities connected with the CEBAF Photon-Tagger project now are centered at the new laboratory (leaving the HARP project and our student-training activities in Washington). We also have been promised additional laboratory and office space to aid in this endeavor.

Next month (July 22-24) we are hosting the Hall B (CLAS) Collaborative Meeting at our Virginia Campus. Among other things, this will be an opportunity for our students (and others in the Washington-area nuclear physics community) to be exposed to the whole range of physics and instrumentation being planned for Hall $B$ at $C E B A F$.

\section{B. The GWU Center for Nuclear Studies}

The Center for Nuclear Studies is the organizational focus for most of our research-related activities, and continues to be supported by GWU as one of its "Centers of Excellence" within the University. Earlier this year, the three-year funding of the Center was renewed (and augmented somewhat) for 1993-96. Through the Center, we derive funding for our Electro-Mechanical Technician and two Graduate Research Assistants. Under its auspices, we not only administer the Nuclear Detector Laboratories, but we hold our Friday Bag Lunch Seminars as well, which have, over the years, become a popular fixture of the Washington-area nuclear physics community.

\section{Junior Personnel Supported by This Grant}

The grant currently supports two postdoctoral fellows and five graduate students (three for the summer only). Dr. P.L. Cole is primarily engaged in CEBAF-related activities, including the physics of high-energy photoreactions and a variety of calculations of event generators and acceptance functions for the CLAS, and also is in charge of the wire-chamber design and construction for the HARP. Dr. S.L. Rugari is primarily engaged with the HARP project, including the physics of the $\left(e, e^{\prime} n\right)$ reaction, the ${ }^{12} \mathrm{C}(n, p)$ experiments conducted at LAMPF/WNR during the summers of 1991 and 1992, and Monte-Carlo calculations in support of the HARP, and also is involved with the PPAD design for the photofission experiment, the tagged-coherentbremsstrahlung work, and the installation and mapping of the tagger magnet at CEBAF. Mr. J.C. Sanabria and Ms. C. Cetina are currently performing scintillator light-collection studies (with the aid of GUIDE7) and Mr. S.A. Philips is doing Monte-Carlo simulation studies (with 
GEANT) for the HARP project; Mr.D.Pang and Mr. K.R. Hossain are working on the photomultiplier tubes and bases for the focal-plane detector array for the photon tagger, and Mr. Pang will spend the second half of the summer at Bates in preparation for our upcoming ${ }^{21} \mathrm{Ne} / 23 \mathrm{Na}$ experiment there, part of which is slated to become his $\mathrm{Ph} . \mathrm{D}$. thesis project. Cetina, Philips, Pang, and Hossain attended the 1993 HUGS at CEBAF. Ms. R.W. Caress achieved her Master's degree last year and is now working at Los Alamos; her M.S. thesis was on Monte-Carlo calculations for the HARP. Two of our undergraduate physics majors, Mr. P. Jordan and Mr. S.M. Wennersten, have participated in work at the NDL; Mr. Wennersten also participated in the WNR experiments, and was invited to deliver a paper on his work at the $7^{\text {th }}$ National Conference on Undergraduate Research and publish it in the Proceedings (see Appendix). 
* = Reproduced in the Appendix

\section{A. Journal Articles}

1. K.G. McNeill, M.N. Thompson, A.D. Bates, J.W. Jury, and B.L. Berman Isospin Effects in the Photodisintegration of Light Nuclei

Phys. Rev. C 47, 1108 (1993)

2. Z. Zhao, R.H. France III, K.S. Lai, S.L. Rugari, M. Gai, and E.L. Wilds The Astrophysical S-Factor of ${ }^{12} \mathrm{C}(\alpha, \gamma){ }^{16} \mathrm{O}$ from the Beta-Delayed Alpha-Particle Emission of $16 \mathrm{~N}^{*}$

Phys. Rev. Lett. 70, 2066; (E) 3524 (1993)

3. T. Alexopoulos et al.

Mass Identified Particle Production in Proton-Antiproton Collisions at $\mathrm{s}^{1 / 2}=300,540$, 1000 , and $1800 \mathrm{GeV}$

Phys. Rev. D (in press)

4. T. Alexopoulos et al.

A Study of Source Size in Proton-Antiproton Collisions at $\mathrm{s}^{1 / 2}=1.8 \mathrm{TeV}$ Using Pion Interferometry

Phys. Rev. D (in press)

5. S.L. Rugari, R.H. France III, B.J. Lund, Z. Zhao, and M. Gai Broken Reflection Symmetry and Isospin Dependence of Enhanced E1 Decays in ${ }^{114} \mathrm{Xe}$ Phys. Rev. C (submitted)

6. T. Alexopoulos et al.

Inclusive Photon Production from Proton-Antiproton Collisions at $\mathrm{s}^{1 / 2}=1.8 \mathrm{TeV}$

Phys. Rev. Lett. (submitted)

\section{B. Papers in Conference Proceedings}

7.* $\quad$ S.M. Wennersten

Large-Angle Protons from Neutron Bombardment of Carbon

Proc. $7^{\text {th }}$ Nat. Conf. Undergrad. Res., Salt Lake City (1993) 


\section{Abstracts}

8. B.M.K. Nefkens, G.C. Anderson, D.B. Barlow, B.L. Berman, W.J. Briscoe, A. Mokhtari, A.M. Petrov, C. Pillai, and M.E. Sadler Comparison of Pion and Electron Inelastic Scattering on ${ }^{3} \mathrm{H}$ and ${ }^{3} \mathrm{He}$ Close to Breakup Bull. Am. Phys. Soc. 37, 1303 (1992)

9. M. McKinzie, M. Burlein, H.T. Fortune, E. Insko, M. Kagarlis, P. Kutt, G. Liu, J.M. O'Donnell, G. Ramussen, J.D. Silk, S. Mordechai, and K.S. Dhuga Inelastic Pion Scattering from ${ }^{27} \mathrm{Al},{ }^{28} \mathrm{Si}$ Bull. Am. Phys. Soc. 37, 1303 (1992)

10. B.L. Berman

Pion Scattering from Very Light Nuclei

Bull. Am. Phys. Soc. 38, 944 (1993)

11. C.M. Laymon, W. Amos, M.G. Burlein, H.T. Fortune, T. Ekenberg, A. Katwal, J.M. O'Donnell, J.D. Silk, J. Zumbro, C.L. Morris, S.J. Seestrom, K.S. Dhuga, R. Garnett, M. Rawool, C.F. Moore, S.L. Morris, and D. Watson

Neutron and Proton Transition Matrix Elements of $58,60,62,64 \mathrm{Ni}$ from Pion Inelastic Scattering Bull. Am. Phys. Soc. 38, 944 (1993)

12. D.M. Manley et al. Inelastic Electron Scattering from ${ }^{18} \mathrm{O}$ Bull. Am. Phys. Soc. (in press)

\section{Proposals}

13.* K.S. Dhuga et al.

Recoil Polarization of the Neutron in the Reactions ${ }^{3} \mathrm{He}\left(e, e^{\prime} n\right)$ and ${ }^{4} \mathrm{He}\left(e, e^{\prime} n\right)$ NIKHEF Proposal 93-09

14.* B.L. Berman et al. Photoreactions on ${ }^{3} \mathrm{He}$ CEBAF Proposal 93-044

15.* N. Bianchi et al. Photoabsorption and Photofission of Nuclei CEBAF Proposal 93-019

16.* B.L. Berman et al. Vector Meson Production from the Proton and Deuteron CEBAF Letter of Intent 93-006 


\section{E. Reports}

17.* P.L. Cole

A Generalized FASTMC for the CLAS

CLAS Note 93-006

18.* J. Ballon, K. Grüner, G. Laborie, A. Pelissier, F. Vezzu, E. Voutier, P.L. Cole, and J.C. Sanabria

The Design and Construction of the HARP MWPCs

HARP Note, April, 1993

19.* E. Voutier, K. Grüner, and P.L. Cole

Optimization of the HARP MWPC Design

HARP Internal Report, April, 1993

20. R.W. Caress

Application of Monte-Carlo Techniques to the Design of a High-Acceptance Recoil Polarimeter

M.S. thesis, The George Washington University (1993) 
Table I. Experiments Planned for the Coming Year

\begin{tabular}{lllll}
\hline Reaction & Laboratory & Physics Emphasis & Status & Reference \\
\hline${ }^{21} \mathrm{Ne},{ }^{23} \mathrm{Na}\left(\mathrm{e}, \mathrm{e}^{\prime}\right)$ & Bates* & Collective excitations & Planned for early 1994 & 1 \\
${ }^{1} \mathrm{H}\left(\pi^{-}, \pi^{\circ}\right) \mathrm{n}$ & LAMPF & Absolute cross section & Scheduled for Aug. 1993 & 2 \\
${ }^{4} \mathrm{He}\left(\mathrm{e}, \mathrm{e}^{\prime} n\right)$ & NIKHEF & Longitudinal-transverse separation & Planned for Fall, 1993 & 3 \\
\hline
\end{tabular}

${ }^{*}$ Spokesperson or co-spokesperson 
Table II. Future Experiments

\begin{tabular}{|c|c|c|c|c|}
\hline Reaction & Laboratory & Physics Emphasis & Status & Reference \\
\hline${ }^{4} \mathrm{He}\left(\mathrm{e}, \mathrm{e}^{\prime} n\right)$ & NIKHEF* & Final-state interactions & Approved, 1993 & 4 \\
\hline${ }^{3} \mathrm{He}\left(\mathrm{e}, \mathrm{e}^{\prime} \mathrm{pn}\right)$ & NIKHEF & Two-body correlations & Approved, 1991 & 5 \\
\hline${ }^{3} \mathrm{He}+\gamma$ & CEBAF* & $\begin{array}{l}\text { Resonances in nuclei; three-body forces; } \\
\Delta \text { knockout }\end{array}$ & Approved, 1993 & 6 \\
\hline$A(\gamma$, tot $),(\gamma, f)$ & CEBAF* $^{*}$ & $\begin{array}{l}\text { Damping of resonances in heavy nuclei; } \\
\text { total photon absorption }\end{array}$ & Approved, 1993 & 7 \\
\hline${ }^{1} \mathrm{H},{ }^{3} \mathrm{He}(\gamma, \phi),(\gamma, \rho)$ & CEBAF & Hidden color & Approved, 1993 & 8 \\
\hline${ }^{3} \mathrm{He}(\gamma, \mathrm{K})$ & CEBAF & Strangeness production on nuclei & Approved, 1991 & 9 \\
\hline${ }^{1} \mathrm{H}(\gamma, \eta),\left(\gamma, \eta^{\prime}\right)$ & CEBAF & $\eta$ and $\eta^{\prime}$ production & Approved, 1991 & 10 \\
\hline${ }^{1} \mathrm{H}\left(\mathrm{e}, \mathrm{e}^{\top} \mathrm{N}\right) \pi$ & CEBAF & $\Delta$ deformation; the Roper resonance & Deferred, 1993 & 11 \\
\hline $1,2 \mathrm{H}(\gamma, \rho),(\gamma, \omega),(\gamma, \phi)$ & CEBAF* & Vector-meson production & Letter of Intent, 1993 & 12 \\
\hline
\end{tabular}

*Spokesperson or co-spokesperson 
Table III. Recent Studies of Few-Body Nuclei with Electromagnetic and Hadronic Probes

\begin{tabular}{|c|c|c|c|c|}
\hline Reaction & Laboratory & Physics Emphasis & Status & Reference \\
\hline${ }^{1} H(n, p)$ & LAMPF-WNR* & Application to HARP & Data being analyzed & 13 \\
\hline${ }^{3} \mathrm{H},{ }^{3} \mathrm{He}\left(\pi^{ \pm}, \pi^{ \pm}\right)$ & LAMPF-EPICS* & $\begin{array}{l}180-\text { to } 295-\mathrm{MeV} \text { cross sections, form } \\
\text { factors, charge asymmetry, spin-flip } \\
\text { amplitudes }\end{array}$ & $\begin{array}{l}\text { Data analyzed, two papers in } \\
\text { preparation }\end{array}$ & 15 \\
\hline${ }^{3} \mathrm{H},{ }^{3} \mathrm{He}\left(\pi^{ \pm}, \pi^{ \pm}\right)$ & LAMPF-EPICS* & $\begin{array}{l}180^{\circ} \text { cross sections, } 142 \text { to } 256 \mathrm{MeV} \text {, } \\
\text { form factors, non-spin-flip amplitudes }\end{array}$ & $\begin{array}{l}\text { Data analyzed, paper in } \\
\text { preparation }\end{array}$ & 16 \\
\hline${ }^{3} \mathrm{H},{ }^{3} \mathrm{He}\left(\pi^{ \pm}, \pi^{ \pm}\right)$ & LAMPF-EPICS* & $\begin{array}{l}180-\mathrm{MeV} \text { cross sections and angular } \\
\text { distribution, charge asymmetry }\end{array}$ & $\begin{array}{l}\text { Data analyzed, paper in } \\
\text { preparation }\end{array}$ & 17 \\
\hline${ }^{6} \mathrm{Li}\left(\mathrm{e}, \mathrm{e}^{\prime} \mathrm{t}\right)$ & NIKHEF* & $\begin{array}{l}\text { Momentum distributions, reaction } \\
\text { mechanism, q-dependence }\end{array}$ & $\begin{array}{l}\text { Data analyzed, paper in } \\
\text { preparation }\end{array}$ & 19 \\
\hline${ }^{6} \mathrm{Li}\left(\mathrm{e}, \mathrm{e}^{\prime} \tau\right)$ & NIKHEF* & $\begin{array}{l}\text { Momentum distributions, reaction } \\
\text { mechanism, validity of } 2 \text {-body model }\end{array}$ & $\begin{array}{l}\text { Data being analyzed, two } \\
\text { papers in preparation }\end{array}$ & 20 \\
\hline${ }^{6} \mathrm{Li}\left(\pi^{+}, 2 p\right)$ & PSI & $\begin{array}{l}\text { Energy dependence near the delta } \\
\text { resonance }\end{array}$ & $\begin{array}{l}\text { Data analyzed, paper in } \\
\text { preparation }\end{array}$ & 21 \\
\hline
\end{tabular}

* Spokesperson or co-spokesperson 
Table IV. Recent Studies of Selected Shell-Model Nuclei with Electromagnetic and Hadronic Probes

\begin{tabular}{|c|c|c|c|c|}
\hline Reaction & Laboratory & Physics Emphasis & Status & Reference \\
\hline${ }^{12} C\left(e, e^{\prime} d\right)$ & NIKHEF & Spectral functions, q-dependence & $\begin{array}{l}\text { Data analyzed, one paper } \\
\text { published, one in preparation }\end{array}$ & 22 \\
\hline${ }^{12} C(n, p)$ & LAMPF-WNR* & Application to HARP & Data being analyzed & 23 \\
\hline${ }^{12} \mathrm{C},{ }^{16} \mathrm{O},{ }^{40} \mathrm{Ca}\left(\pi^{ \pm}, \pi^{ \pm}\right)$ & LAMPF-EPICS* & $\begin{array}{l}\text { Excitation functions at large angles, } \\
\text { medium modifications }\end{array}$ & $\begin{array}{l}\text { Data analyzed, two papers } \\
\text { published, one in preparation }\end{array}$ & 24 \\
\hline $\begin{array}{l}{ }^{12} \mathrm{C},{ }^{16} \mathrm{O},{ }^{40} \mathrm{Ca},{ }^{48} \mathrm{Ca} \\
{ }^{90} \mathrm{Zr}_{,}{ }^{208} \mathrm{~Pb}\left(\pi^{ \pm}, \pi^{ \pm}\right) \\
\left(\pi^{ \pm}, \pi^{ \pm}\right)\end{array}$ & LAMPF-P $3 *$ & $\begin{array}{l}\text { Exploratory }(300-500 \mathrm{MeV}) ; \text { test of } \\
\text { optical models }\end{array}$ & $\begin{array}{l}\text { Data being analyzed, invited } \\
\text { paper presented, paper in } \\
\text { preparation }\end{array}$ & 25 \\
\hline${ }^{16} \mathrm{O}\left(\mathrm{e}, \mathrm{e}^{\prime}\right)$ & Bates & High-excitation, isovector states & $\begin{array}{l}\text { Data analyzed, paper in } \\
\text { preparation }\end{array}$ & 26 \\
\hline${ }^{16} \mathrm{O}\left(\pi^{+}, \pi^{-}\right)^{16} \mathrm{Ne}$ & LAMPF-EPICS* & $\begin{array}{l}\text { Interference effects in double charge } \\
\text { exchange }\end{array}$ & Data being analyzed & 27 \\
\hline $17,180\left(p, p^{\prime}\right)$ & IUCF & $\begin{array}{l}135-\mathrm{MeV} \text { cross sections, comparison } \\
\text { with }\left(e, \mathrm{e}^{\prime}\right)\end{array}$ & $\begin{array}{l}\text { Data analyzed, two papers in } \\
\text { preparation }\end{array}$ & 28 \\
\hline${ }^{18} \mathrm{O}\left(\mathrm{e}, \mathrm{e}^{\prime}\right)$ & Bates & Stretched-spin states; isospin structure & $\begin{array}{l}\text { Data analyzed, paper in } \\
\text { preparation }\end{array}$ & 29 \\
\hline${ }^{30} \mathrm{Si}\left(\mathrm{e}, \mathrm{e}^{\prime}\right)$ & Bates* & Stretched-spin states & $\begin{array}{l}\text { Data analyzed, paper in } \\
\text { preparation }\end{array}$ & 30 \\
\hline$A(\gamma, n$ and $p)$ & LLNL, Melbourne & Isospin Structure of the GDR & Published & 31 \\
\hline
\end{tabular}

* Spokesperson or co-spokesperson 


\section{REFERENCES TO TABLES}

1. D. M. Manley et al., Bates Proposal 88-18

2. M. E. Sadler et al., LAMPF Proposal 1268

3. T. S. Bauer et al., NIKHEF Proposal 91-01

4. K. S. Dhuga et al., NIKHEF Proposal 93-09

5. T. S. Bauer et al., NIKHEF Proposal 91-02

6. B. L. Berman et al., CEBAF Proposal 93-044

7. N. Bianchi et al., CEBAF Proposal 93-019

8. J.-M. Laget et al., CEBAF Proposal 93-031

9. C. E. Hyde-Wright et al., CEBAF Proposal 91-014

10. B. G. Ritchie et al., CEBAF Proposal 91-008

11. J. J. Kelly et al., CEBAF Proposal 93-013

12. B. L. Berman et al., CEBAF Letter of Intent 93-006

13. B.L. Berman et al., LAMPF Proposal 4N0041 (1991)

14. C. Pillai et al., Phys. Lett. 207B, 389 (1988); C. Pillai et al., Phys. Rev. C 43, 1838 (1991); B.L. Berman et al., to be submitted to Phys. Rev. C

15. K.S. Dhuga et al., and C. Pillai et al., to be submitted to Phys. Rev. C; see K.S. Dhuga et al., Proc. Particles and Nuclei Int. Conf. (1990) and B.L. Berman et al., Proc. Int. Workshop on Pions in Nuclei (1991)

16. S.K. Matthews et al., Bull. Am. Phys. Soc. 35, 945 (1990) and to be submitted to Phys. Rev. C

17. S.K. Matthews et al., to be submitted to Phys. Rev. C; see S.K. Matthews, et al., S.J.Greene et al., and W.J. Briscoe et al., Proc. Int. Conf. on Nuclear Physics (1992) 
18. R. Ent et al., Phys. Rev. Lett. 57, 2367 (1986); R. Ent et al., Proc. Particles and Nuclei Int. Conf. and Verhandl. Deutsche Phys. Ges. (1990), and to be submitted to Phys. Rev. C

19. D. Zubanov et al., Bull. Am. Phys. Soc. 35, 927 (1990) and to be submitted to Phys. Rev. C

20. B.L. Berman et al. and A. Mokhtari et al., to be submitted to Phys. Rev. C; see B.L. Berman et al., NIKHEF Proposal 88-E1 (1987)

21. D. Zhang et al., Bull. Am. Phys. Soc. 35, $946(1990)$ and to be submitted to Phys. Rev. C

22. R. Ent et al., Phys. Rev. Lett. 62, 24 (1989); R. Ent et al., to be submitted to Phys. Rev. C; also see R. Ent et al., in Proc. CEBAF 1988 Summer Workshop

23. S.M. Wennersten, Proc. $7^{\text {th }}$ Nat. Conf. Undergrad. Res., Salt Lake City, 1993; also see Ref. 13

24. K.S. Dhuga et al., Phys. Rev. C 32, 2208 (1985); K.S. Dhuga et al., Phys. Rev. C 35, 1148 (1987); also see K.S. Dhuga et al., LAMPF Proposal 1018 (1986)

25. K.S. Dhuga et al., LAMPF Proposal 1106 (1987); K.S. Dhuga et al., invited paper at Workshop on Pion Scattering Above the Delta Resonance (KEK, Japan, 1990)

26. C.E. Hyde-Wright et al., to be submitted to Phys. Rev. C

27. K.S. Dhuga et al., LAMPF Proposal 1017 (1986)

28. J. Kelly et al., Proc. Workshop Nuclear Structure with Intermediate Energy Probes (Los Alamos, 1980), p. 461 and to be submitted to Phys. Rev. C

29. R.M. Sellers et al., Bull. Am. Phys. Soc. 35, 927 (1990) and to be submitted to Phys. Rev. C; D.M. Manley et al., Bull. Am. Phys. Soc. (in press)

30. L. Knox et al., Bull. Am. Phys. Soc. 34, 1812 (1989) and to be submitted to Phys. Rev. C

31. K.G. McNeill et al., Phys Rev. C 47, 1108 (1993) 